\title{
The structural and scaling properties of nearby galaxy clusters
}

\section{The universal mass profile ${ }^{\star}$}

\author{
E. Pointecouteau ${ }^{1}$, M. Arnaud ${ }^{1}$, and G. W. Pratt ${ }^{2}$ \\ ${ }^{1}$ CEA/DSM/DAPNIA Service d'Astrophysique, CE Saclay, L'Orme des Merisiers, Bât. 709, 91191 Gif-sur-Yvette, France \\ e-mail: pointeco@discovery.saclay.cea.fr \\ 2 MPE, Giessenbachstraße, 85748 Garching, Germany
}

Received 17 December 2004 / Accepted 21 January 2005

\begin{abstract}
We present the integrated mass profiles for a sample of ten nearby $(z \lesssim 0.15)$, relaxed galaxy clusters, covering a temperature range of [2-9] keV, observed with XMM-Newton. The mass profiles were derived from the observed gas density and temperature profiles under the hypothesis of spherical symmetry and hydrostatic equilibrium. All ten mass profiles are well described by an NFW-type profile over the radial range from 0.01 to $0.5 R_{200}$, where $R_{200}$ is the radius corresponding to a density contrast of 200 with respect to the critical density at the cluster redshift. A King model is inconsistent with these data. The derived concentration parameters and total masses are in the range $c_{200}=4-6$ and $M_{200}=1.2 \times 10^{14}-1.2 \times 10^{15} M_{\odot}$, respectively. Our qualitative and quantitative study of the mass profile shape shows, for the first time, direct and clear observational evidence for the universality of the total mass distribution in clusters. The mass profiles scaled in units of $R_{200}$ and $M_{200}$ nearly coincide, with a dispersion of less than $15 \%$ at $0.1 R_{200}$. The $c_{200}-M_{200}$ relation is consistent with the predictions of numerical simulations for a $\Lambda \mathrm{CDM}$ cosmology, taking into account the measurement errors and expected intrinsic scatter. Our results provide further strong evidence in favour of the Cold Dark Matter cosmological scenario and show that dark matter collapse is well understood, at least down to the cluster scale.
\end{abstract}

Key words. cosmology: observations - cosmology: dark matter - X-rays: galaxies: clusters - galaxies: clusters: general

\section{Introduction}

The Dark Matter (DM) distribution in clusters is a sensitive test of current scenarios of structure formation and of the nature of the DM itself. Of particular interest are comparisons with the predictions of $N$-body simulations of hierarchical clustering in the currently favoured $\Lambda$ CDM (Cold Dark Matter) cosmology. Numerical simulations (e.g Navarro et al. 1997), as well as early semi-analytical work (e.g. Bertschinger 1985), predict a remarkable similarity in the Cold Dark Matter density profile of virialized halos. Although the exact slope in the centre is still a matter of debate, recent high resolution simulations predict that dark matter profiles are cusped (Navarro et al. 1997; Moore et al. 1999; Diemand et al. 2004; Navarro et al. 2004) and that the concentration of the Dark Matter varies only slightly with system mass (e.g. Dolag et al. 2004).

The strong similarity in the ROSAT surface brightness profiles (Vikhlinin et al. 1999; Neumann \& Arnaud 2001; Arnaud et al. 2002a), and of the temperature profiles of hot clusters observed with ASCA and BeppoSAX (Markevitch et al. 1998; Irwin \& Bregman 2000; De Grandi \& Molendi 2002) provided indirect evidence of a universal underlying dark

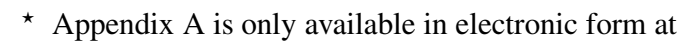
http://www. edpsciences.org matter distribution. The present generation of X-ray satellites, XMM-Newton and Chandra, represent a giant step forward in terms of resolution and sensitivity. We can now measure precisely, through the hydrostatic equilibrium equation, the total mass distribution in clusters. Evidence is slowly accumulating that CDM numerical simulations predict the correct shape of the Dark Matter distribution, not only in massive clusters (e.g. David et al. 2001; Allen et al. 2001; Arabadjis et al. 2002; Buote \& Lewis 2004; Buote 2004; Allen et al. 2003), but also in low mass clusters (Pratt \& Arnaud 2003). This may well be true up to the virial radius, as shown up to $\delta=500$ by the observation of A1413 (Pratt \& Arnaud 2002). The observed profiles are cusped in the centre, and, for a few massive clusters, the inner slope has even been measured precisely enough to distinguish between various CDM predictions (Lewis et al. 2003; Buote \& Lewis 2004; Pointecouteau et al. 2004). However, most mass studies have been conducted on individual "test case" clusters. Recently, Pratt \& Arnaud (2005) performed the first quantitative check of the universality of the mass profile using a sample of five clusters observed with XMM-Newton (four low mass systems compared to one massive system). It is necessary to extend this type of study to larger samples, with a better temperature (i.e., mass) coverage. 
In this paper, we use XMM-Newton to examine the total mass profile of ten relaxed, nearby clusters in the temperature range from 2 to $9 \mathrm{keV}$. In a companion paper (Arnaud et al. 2005; Paper II), we use the mass data to study the scaling properties of the mass with temperature. In Sect. 2 we present the sample, the observations and the data processing steps. We detail the extraction of the scientific products, from temperature and density profiles to mass profiles. We quantify the shape of the mass profiles in Sect. 3. Our results are discussed and we conclude in Sect. 4.

We have used the currently favoured $\Lambda \mathrm{CDM}$ cosmology, $H_{0}=70 \mathrm{~km} \mathrm{~s}^{-1} \mathrm{Mpc}^{-1}, \Omega_{m}=0.3$ and $\Omega_{\Lambda}=0.7$, throughout this paper.

\section{XMM-Newton observations and data processing}

\subsection{The sample}

The sample has been built to cover a wide range in temperature (i.e. in mass) from 2 to $9 \mathrm{keV}$, and constitutes 10 clusters. We limited the redshift range to $z \leq 0.15$, where evolution effects are expected to be negligible. With the exception of A478 and PKS0745, for which there was a mosaic observation, we only considered clusters fitting in the XMM-Newton field of view, enabling the local background to be estimated, thus limiting systematic uncertainties on the temperature, and consequently, the mass profiles. Since cluster size increases with temperature, this last criterion sets a lower limit on the redshift for each temperature. Cool and hot clusters lie in a redshift range close to this limit $([0.04,0.06]$ and $[0.1-0.15]$ respectively). This thus optimizes both the cluster coverage and the statistical quality of the data. A final selection criterion was the quality of the mass data. All clusters in the sample have a regular X-ray morphology, indicative of a relaxed state and allowing reliable determination of the total mass profile through the hydrostatic equilibrium equation.

Our sample includes the sample of Pratt \& Arnaud (2005): the cool clusters A1983, A1991, MKW9, A2717, and the hot cluster A1413. We improved the temperature coverage by adding A478 (recently studied by Pointecouteau et al. 2004), and four clusters at intermediate and high temperature from the $X M M$-Newton archive which meet our criteria. The journal of observations is presented in Table 1.

To minimise systematic errors in the statistical study of cluster properties, it is important to use scientific data derived, as far as possible, with the same procedure. Unless otherwise stated, we use the previously published data of the cool clusters (Pratt \& Arnaud 2003, 2005), A478(Pointecouteau et al. 2004) and A1413(Pratt \& Arnaud 2002) ${ }^{1}$. These results were obtained with the same general method that we use to process the four additional clusters, although some details differ from cluster to cluster. The procedure is described in the next four sections. Further details and comments on each individual target are given in Appendix A.

\footnotetext{
${ }^{1}$ We rescaled the published values of A1983 and A1413 to the $\Lambda \mathrm{CDM}$ cosmology used in this paper.
}

Table 1. Journal of observations.

\begin{tabular}{lcccc}
\hline \hline Cluster & $z$ & Rev. & Mode $^{a}$ & $t_{\text {exp }}(\mathrm{ks})$ \\
\hline A1983 & 0.0442 & 400 & EFF & $18 / 18 / 12$ \\
A2717 & 0.0498 & 558 & FF & $52 / 52 / 44$ \\
MKW9 & 0.0382 & 311 & EFF & $30 / 30 / 21$ \\
A1991 & 0.0586 & 584 & FF & $29 / 29 / 19$ \\
A2597 & 0.0852 & 179 & FF & $16 / 15 / 10$ \\
A1068 & 0.1375 & 633 & FF & $19 / 20 / 15$ \\
A1413 & 0.1430 & 182 & EFF & $24 / 25 / 10$ \\
A478 & 0.0881 & 401,411 & EFF & $48 / 41 / 37^{c}$ \\
PKS 0745 & 0.1028 & 164 & - & $10 / 10 /-^{c}$ \\
A2204 & 0.1523 & 322 & FF & $20 / 20 / 13$ \\
\hline
\end{tabular}

Notes: ${ }^{a}$ EPN observation mode: FF $=$ Full Frame, EFF $=$ Extended Full Frame. ${ }^{b}$ Exposure time (EMOS1/EMOS2/EPN) in ks after flare cleaning. ${ }^{c}$ The exposure times are given for the central pointing.

\subsection{Event list processing}

We made use of the XMM-Newton SAS software package, versions 5.3 or 5.4 , to filter the data. Below we detail the main data processing steps.

1. Considering only events with $F L A G=0$ and PATTERN $\leq$ 12 (EMOS) and PATTERN = $0(\mathrm{EPN})$, we clean the data for soft proton flares using a threshold cut method (see Appendix A in Pratt \& Arnaud 2002). A first screening was performed using light curves in the high energy band ([10-12] keV for EMOS, [12-14] keV for EPN) using $100 \mathrm{~s}$ bins. After filtering using the good time intervals from this screening, a second screening was performed as an extra check. A second light curve built in $10 \mathrm{~s}$ bins in a wider energy band ([0.3-10] keV for EMOS, [0.3-12] keV for EPN) was screened, and the event lists were filtered accordingly.

2. To correct for the vignetting effect, we used the photon weighting method (Arnaud et al. 2001). The weight coefficients were computed by applying the SAS task EVIGWEIGHT to each event file (including background files).

3. The point source lists from the SOC pipeline were visually checked on images generated for each detector. Selected point sources from all available detectors were gathered into a single point source list and the events in the corresponding regions were removed from the event lists. The area lost due to point source exclusion (as well as CCD gaps and bad pixels) was computed using a mask image.

We used XMM-Newton dedicated blank field datasets from either Lumb et al. (2002) or Read \& Ponman (2003) to obtain a background event list associated with each data set $^{2}$.

\footnotetext{
2 Most observations used for the Read \& Ponman (2003) blank field were centred on point sources. Their removal, and the removal of other point sources in each field, produced local variations of a factor of two from point to point in the final stacked exposure map. We took this variation into account in our analysis.
} 
For each cluster, the blank field event list was recast in order to match the astrometry of the observation and was then processed in the same way as the observation event list. (This included extraction of the events in the same regions as the point sources in the observation data set.) To account for variations in the particle background level, we assumed that only particle signal is collected at high energy, and used the ratio between the high energy count rate of the observation and the blank field to renormalise the blank field counts. The bands used were [10-12] keV for EMOS and [12-14] keV for EPN. To avoid the contamination due to possible high energy emission from the cluster, a central region $\left(r<5^{\prime}\right)$ was excluded when computing the normalisation in the case of the hot clusters. For EPN, the out-of-time (OoT) events were considered as an additional background component. For each observation the OoT event list was generated using the SAS task EPCHAIN and processed in the same way as the observation. A normalisation factor was then used depending on the observing mode (Full Frame or Extended Full Frame).

The cleaned event list, the blank field and, in the case of the EPN, the OoT event file of each single observation were used to extract scientific products such as spectra and surface brightness profiles. Background subtraction was performed using the double subtraction process fully described in Arnaud et al. (2002b, Appendix), and involves subtraction of the normalised blank field data, and subsequent subtraction of the Cosmic X-ray background residual estimated from a region free of cluster emission.

\subsection{The density profile}

For each cluster, an azimuthally-averaged, background subtracted surface brightness (SB) profile was computed in the soft energy band ([0.3-2.] keV in the present work) for each available detector ${ }^{3}$. The profiles of all detectors were then summed together into a total SB profile and rebinned with logarithmic radial binning and a minimum $\mathrm{S} / \mathrm{N}$ ratio of $3 \sigma$. The profile was corrected for radial variations of the emissivity (e.g due to abundance or temperature gradients) in the energy band considered (see Pratt \& Arnaud 2003, for details). This corrected profile is thus proportional to the emission measure along the line of sight.

The final SB profiles were fitted using parametric analytic models of the gas density profile, converted to an emission measure profile and convolved with the PSF spatial response (Ghizzardi 2001, 2002). We considered various parametric forms and empirically chose the model best fitting the data using the $\chi^{2}$ statistic as a measure of the goodness of fit. The models included a double $\beta$-model (the BB model defined in Pratt $\&$ Arnaud 2002), a modified double $\beta$-model, which allows a more concentrated gas density distribution towards the centre (the KBB model used by Pratt \& Arnaud 2002 for A1413),

\footnotetext{
3 We also computed background subtracted SB profiles in the high energy band (we recall: [10-12] keV for EMOS and [12-14] keV for EPN) to check that there were no significant residuals, i.e., that the normalisation of the blank field background was correct, and that it was indeed constant with radius.
}

and the sum of three $\beta$-models in which a common value of $\beta$ is assumed to ensure smooth behaviour at large radii (the BBB model used by Pointecouteau et al. 2004 to model the SB profile of A478). For the clusters newly analysed here, the best fitting model was either a KBB model (A2597, A1068 and PKS 0745-191) or a BBB model (A2204). Over the whole sample the reduced $\chi^{2}$ values vary from 1.1 (for A478), to 1.5 (for A1068), and thus even the best parametric model leads in some cases to a formally unacceptable fit. This is linked to the very small statistical errors on each measurement. The actual discrepancies between the model and the data remain small: adding a few percent ( 2 to $5 \%$ ) of systematic error while fitting the data always leads to acceptable $\chi^{2}$ values.

\subsection{The temperature profile}

Concentric annular regions were defined from the total background subtracted SB profile of the cluster the clusters newly analysed here, we used the following empirical method to define the annuli from which the spectra were extracted. We started with a minimum bin size of $15^{\prime \prime}$ (e.g about the HEW of the XMM-Newton PSF) and then increased the bin size by a logarithmic factor of 1.05 . This is sufficient in the central parts to keep a good $\mathrm{S} / \mathrm{N}$, but not in the outer regions. We thus further impose, in the outer regions, that the number of cluster counts per bin is approximately constant, within $5 \%$.

For each annular region, a background subtracted spectrum was extracted for each available detector. The spectra from each detector were then simultaneously fitted using XSPEC (Arnaud 1996) with an absorbed, redshifted single temperature plasma model (WABS*MEKAL). Except in the case of A478 (see Pointecouteau et al. 2004, for details), after checking that the $N_{\mathrm{H}}$ value agreed with the galactic $21 \mathrm{~cm}$ value (from Hartmann \& Burton 1999), this parameter was frozen. Thus we derived an annular temperature profile for each cluster in the sample, as shown in the left panel of Fig. 1. All profiles (except that of A1413) have the same generic shape: a negative gradient towards the centre and a roughly flat external plateau. A1413 is the only cluster for which we observe a significantly decreasing temperature at high radii (of about 20\%).

To derive the true radial temperature profile (needed to compute the mass profile) we should take into account projection and PSF effects. For the typical temperature profile shape we have obtained, these effects depend most strongly on the magnitude of the gradient in the centre. In addition, a strong temperature gradient in the central regions is usually associated with a strongly peaked surface brightness profile, further increasing the PSF blurring ${ }^{4}$. Reconstructing the radial temperature profile in these cases is not a trivial task. General nonparametric methods, such as simultaneous fitting of annular spectra, amplify the noise considerably. This yields radial temperature profiles with unphysically large fluctuations, particularly when both PSF and projection effects are important (see discussion in Pointecouteau et al. 2004).

\footnotetext{
4 The effects of the PSF correction are most noticeable for A2204 and A478.
} 

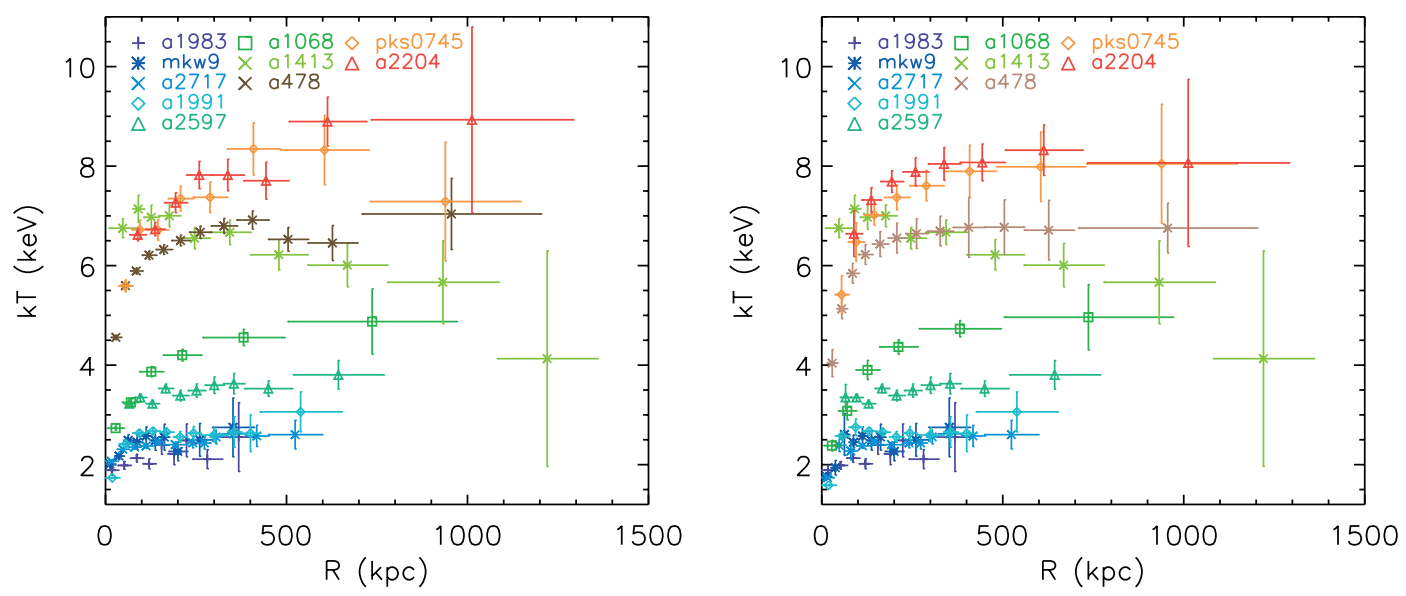

Fig. 1. Left: annular (projected) cluster temperature profiles. Right: deprojected, PSF-corrected temperature profiles.

The clusters newly analysed in the present work have quite strong "cooling flows". We thus applied the method developed for A478 by Pointecouteau et al. (2004), in which the radial temperature profile is derived from the annular temperature profile in the following manner. The noise amplification problem is avoided by using smooth parametric representation of the annular temperature profile. We used the function given by Allen et al. (2001): $T(r)=T_{0}+T_{1}\left[\frac{\left(r / r_{c}\right)^{n}}{1+\left(r / r_{c}\right)^{\eta}}\right]$ to fit the annular profile. The best fitting model profile is then corrected for both the projection and PSF effects, assuming that the annular temperatures are emission weighted temperatures (see Pointecouteau et al. 2004, for details). To estimate the errors we repeated the procedure 1000 times, using a Monte Carlo method that randomizes the annular profile based on the observed errors. Because using a specific functional form effectively limits the allowed profiles, the standard deviation of the corrected temperature at a given point is occasionally smaller than the error on the annular temperature. When this was the case, we kept the observed error.

For the poor systems, which have a more modest central temperature gradient, the radial temperature profile was estimated as described in Pratt \& Arnaud (2003, 2005). For A1413 PSF and projection effects proved to be negligible (Pratt \& Arnaud 2002). The deprojected, PSF corrected profiles of all the clusters are shown in the right panel of Fig. 1. A detailed discussion of the shape of these temperature profiles is beyond the scope of this paper.

\subsection{The total mass profile}

The mass profile for each cluster was derived from the best fitting density profile and the deprojected, PSF corrected temperature profile under the assumptions of hydrostatic equilibrium and spherical symmetry:

$$
M(r)=-\frac{k T r}{\mathrm{G} \mu m_{\mathrm{p}}}\left[\frac{\mathrm{d} \ln n_{\mathrm{g}}}{\mathrm{d} \ln r}+\frac{\mathrm{d} \ln T}{\mathrm{~d} \ln r}\right] .
$$

The mass, and associated errors, were then computed at each radius of the temperature profile ${ }^{5}$ using a Monte Carlo method

\footnotetext{
${ }^{5}$ We use in fact the weighted effective radius of each annulus (Lewis et al. 2003).
}

that randomises the temperature profile based on the observed errors (Pratt \& Arnaud 2003). A cubic spline interpolation is used to compute the temperature derivative at each radius. Only mass profiles which monotonically increase with radius were kept (1000 in total). The errors due to the errors on the density gradients, which are negligible with respect to those from the temperature, were then added. The mass profile of A1413 was originally derived using a slightly different Monte Carlo method (Pratt \& Arnaud 2002). For homogeneity of the present study, we re-derived the mass profile of this cluster with the present method, allowing us to extend the profile to lower radii ${ }^{6}$. The new mass profile is perfectly consistent with the published one.

Each cluster has been checked for the presence of structure such as cold fronts, hot bubbles, ghost cavities, or other effects which could affect the mass determination. Details of each cluster are given in Appendix A.

\section{The shape of the mass profile}

\subsection{Mass profile modelling}

For each cluster, three mass models were fitted to the data: (i) a King isothermal sphere profile; (ii) a standard NFW profile (Navarro et al. 1997); and (iii) an MQGSL profile (Moore et al. 1999). Our data indicate that an isothermal sphere model (i.e., a profile with a core) is not a good representation of the mass distribution in these clusters. Dropping too rapidly in the centre and flattening in the outer regions, the reduced $\chi^{2}$ obtained from King model fits ranged from 1.65 for A478 to 15.6 for A1068. It is rejected with a minimum $91 \%$ confidence level (A478). In contrast, the reduced $\chi^{2}$ obtained from NFW model fits varied from from 0.4 (PKS 0745-191) to 1.8 (A2597),

\footnotetext{
${ }^{6}$ Pratt \& Arnaud (2002) used the original Monte Carlo method of Neumann \& Böhringer (1995). This method uses a "diffusive" process to calculate random temperatures at equally spaced radii. It has two parameters, the radial step and a window parameter, which are optimised to ensure smooth random temperature profiles. In this original version of the code, the radial step fixed the minimum radius of the mass profile. In the present method it is simply the radius of the first temperature annulus.
} 

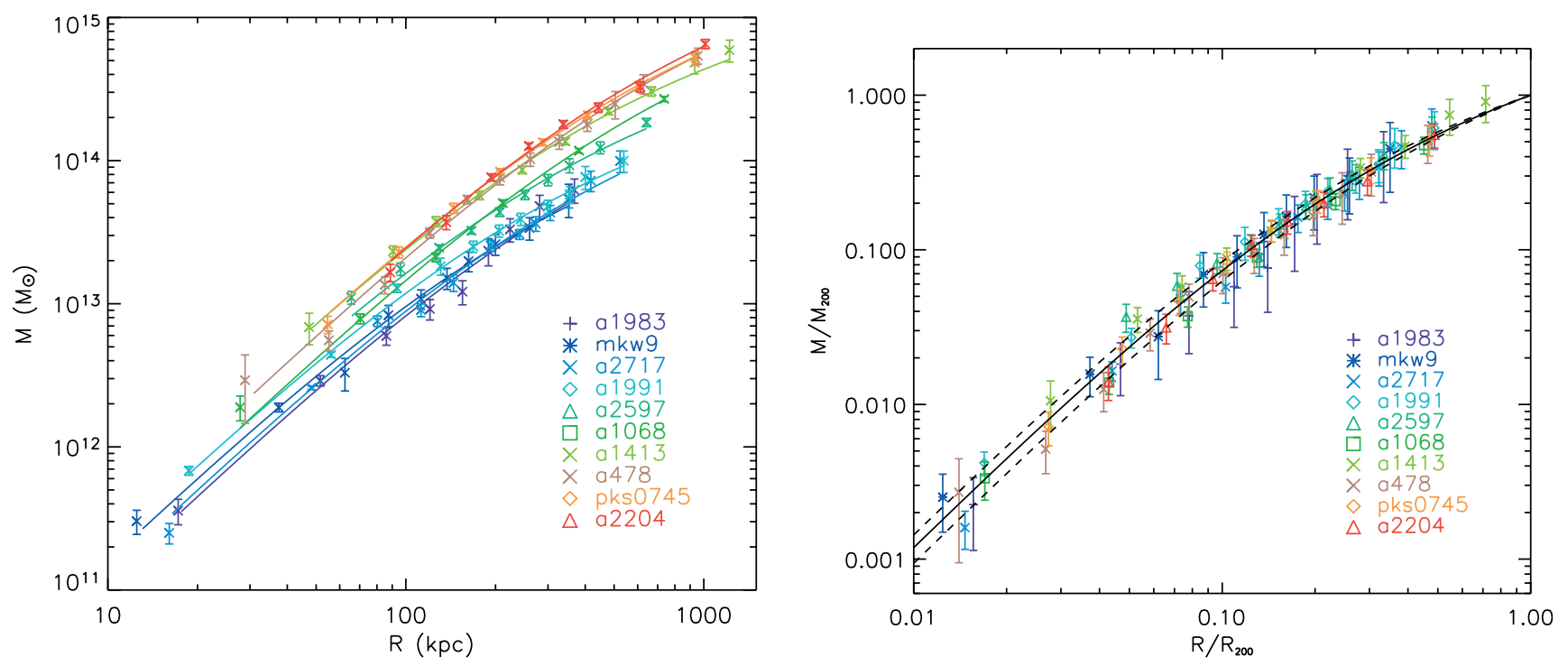

Fig. 2. Left panel: integrated total mass profiles plotted in units of physical radius $(\mathrm{kpc})$. The solid lines are the best fitting NFW profiles as detailed in Table 2. Right panel: scaled mass profiles of all clusters. The mass is scaled to $M_{200}$, and the radius to $R_{200}$, both values being derived from the best fitting NFW model. The solid black line corresponds to the mean scaled NFW profile and the two dashed lines are the associated standard deviation.

Table 2. Results for the NFW mass profile fits.

\begin{tabular}{lccccccc}
\hline \hline Cluster & $c_{200}$ & $R_{200}(\mathrm{kpc})$ & $M_{200}\left(10^{14} M_{\odot}\right)$ & $R_{500}(\mathrm{kpc})$ & $M_{500}\left(10^{14} M_{\odot}\right)$ & $\chi_{\mathrm{NFW}}^{2}(\mathrm{~d} . \mathrm{o.f})$. & {$\left[\chi_{\mathrm{king}}^{2}, \chi_{\mathrm{MQGSL}}^{2}\right]^{a}$} \\
\hline A1983 & $3.83 \pm 0.71$ & $1100 \pm 140$ & $1.59 \pm 0.61$ & $717 \pm 79$ & $1.09 \pm 0.37$ & $9.1(7)$ & {$[31,12]$} \\
MKW9 & $5.41 \pm 0.67$ & $1006 \pm 84$ & $1.20 \pm 0.30$ & $668 \pm 51$ & $0.88 \pm 0.20$ & $3.6(8)$ & {$[24,5.2]$} \\
A2717 & $4.21 \pm 0.25$ & $1096 \pm 44$ & $1.57 \pm 0.19$ & $717 \pm 26$ & $1.10 \pm 0.12$ & $16(10)$ & {$[48,52]$} \\
A1991 & $5.78 \pm 0.35$ & $1106 \pm 41$ & $1.63 \pm 0.18$ & $737 \pm 25$ & $1.20 \pm 0.12$ & $10(9)$ & {$[82,33]$} \\
A2597 & $5.86 \pm 0.50$ & $1344 \pm 49$ & $3.00 \pm 0.33$ & $897 \pm 29$ & $2.22 \pm 0.22$ & $14.6(8)$ & {$[55,6.8]$} \\
A1068 & $3.69 \pm 0.26$ & $1635 \pm 47$ & $5.68 \pm 0.49$ & $1060 \pm 26$ & $3.87 \pm 0.28$ & $2.5(4)$ & {$[62,9.8]$} \\
A1413 & $5.82 \pm 0.50$ & $1707 \pm 57$ & $6.50 \pm 0.65$ & $1129 \pm 33$ & $4.82 \pm 0.42$ & $8.0(8)$ & {$[45,3.7]$} \\
A478 & $4.22 \pm 0.39$ & $2060 \pm 110$ & $10.8 \pm 1.8$ & $1348 \pm 64$ & $7.57 \pm 1.11$ & $9.5(10)$ & {$[16,30]$} \\
PKS 0745 & $5.12 \pm 0.40$ & $1999 \pm 77$ & $10.0 \pm 1.2$ & $1323 \pm 45$ & $7.27 \pm 0.75$ & $2.3(6)$ & {$[20,20]$} \\
A2204 & $4.59 \pm 0.37$ & $2075 \pm 77$ & $11.8 \pm 1.3$ & $1365 \pm 44$ & $8.39 \pm 0.81$ & $9.7(6)$ & {$[14,21]$} \\
\hline
\end{tabular}

${ }^{a}$ Chi-square obtained for the best fit of a king and a MQGSL model respectively (see Sect. 3.1).

while the MQGSL profile yielded reduced $\chi^{2}$ of 0.5 (A1413) to 5.21 (A2717). We note that the chi-squared value is very sensitive to the central points. In some cases, the mass errors on these points may be underestimated due to the procedure used for PSF and projection effects correction, or to systematic errors we are not able to quantify and are therefore unable to take into account.

In all cases except A1413 (Pratt \& Arnaud 2002) and A2597, the NFW profile proved to be a better fit than the MQGSL model, and de facto to be the best representation of our current data. In the cases of A1413 and A2597, the improvement in $\chi^{2}$ when an MQGSL profile is used is very small. Thus, to keep our approach coherent, we decided to use the NFW fit as a parametric representation of the mass profile of each cluster.

The NFW model, where the density is $\rho(r) \propto\left[\left(r / r_{\mathrm{s}}\right)(1+\right.$ $\left.\left.r / r_{\mathrm{s}}\right)^{2}\right]^{-1}$, has two free parameters: the scaling radius $r_{\mathrm{s}}$, and a normalisation parameter. The model can be equivalently expressed in terms of the concentration parameter $c_{200}=r_{200} / r_{\mathrm{s}}$ and the total mass $M_{200} . M_{200}$ is the mass corresponding to a density contrast of $\delta=200$, i.e. the mass contained in a sphere of radius $R_{200}$, which encompasses a mean density of 200 times the critical density at the cluster redshift: $\rho_{\mathrm{c}}(z)=$ $3 E(z)^{2} \mathrm{H}_{0}^{2} / 8 \pi \mathrm{G}$, where $E^{2}(z)=\Omega_{\mathrm{m}}(1+z)^{3}+\Omega_{\Lambda}$. In numerical simulations, this sphere is found to correspond roughly to the virialised part of clusters. The results of the best NFW fits are detailed in Table 2, and the best fitting profiles are shown in Fig. 2.

\subsection{Scaled mass profiles}

The left panel of Fig. 2 shows the mass profiles (and bestfitting NFW models) plotted in physical units (kpc). Not surprisingly, there is a continuous increase in mass with cluster 
temperature, reflecting the temperature coverage of the sample. These unscaled mass profiles already show signs of an underlying similarity in the matter distribution. The right panel of Fig. 2 shows the scaled mass profiles, where we express the radius in terms of $R_{200}$ and the mass in terms of $M_{200}$, these values being derived from the best fitting NFW model of each cluster. The scaled mass profiles cover a wide range of radii, from about $0.01 R_{200}$ to $0.7 R_{200}$, and are particularly well constrained between $0.1 R_{200}$ and $0.5 R_{200}$. The agreement between the scaled profiles is remarkable, reflecting the similarity in shape of the profiles. The average of all best fitting NFW models is shown as a black line, with dashed lines representing the mean plus or minus the standard deviation. The dispersion is small and virtually identical to that derived by Pratt \& Arnaud (2005) from their smaller sample: we obtained a dispersion of $7.4 \%$ at $0.3 R_{200}$ and $14.3 \%$ at $0.1 R_{200}$ (compared to the $8 \%$ and $15 \%$ found by those authors).

\subsection{Variation of the concentration parameter with mass}

Structure formation models do not in fact predict a strictly universal matter distribution in clusters. A weak variation in the concentration is expected from low to high mass clusters, reflecting differences in the formation epochs of low and high mass haloes (Navarro et al. 1997; Bullock et al. 2001; Dolag et al. 2004). Building on the work of Pratt \& Arnaud (2005, their Fig. 12), we can investigate the relation between the concentration parameter and the cluster mass using our extended sample. Figure 3 presents the $c_{200}-M_{200}$ relation found by Dolag et al. (2004) $(1+z) \bar{c}=c_{0}\left(M / M_{0}\right)^{\alpha}$ with $\alpha \sim-0.1$ in a $\Lambda \mathrm{CDM}$ cosmology with $\sigma_{8}=0.9$. All ten points agree with the predicted relation, taking into account its intrinsic dispersion and the measurement errors.

We then performed a linear regression fit in the $\log c_{200}-\log M_{200}$ plane, taking into account the uncertainties on each quantity. The resulting slope of $\alpha=-0.04 \pm 0.03$ $\left(\chi^{2}\right.$ (d.o.f.) $\left.=42(8)\right)$ is poorly constrained, due in part to the limited size of the sample and the large relative uncertainties in the concentration parameter. The result is however compatible with the intrinsic dispersion of theoretical predictions. The best fitting $c(M)$ relation is shown as the long dashed line in Fig. 3.

\section{Discussion and conclusion}

We have measured the total mass profile of ten clusters from $0.01 R_{200}$ up to $0.5 R_{200}$ using $X M M$-Newton observations. Our sample has an excellent temperature coverage and covers an order of magnitude in mass from $M_{200}=1.2 \times 10^{14} M_{\odot}$ to $1.2 \times 10^{15} M_{\odot}$. Our study confirms previous XMM-Newton and Chandra studies conducted on individual targets (see Sect. 1), and extends the initial statistical study of cluster mass profile structure by Pratt \& Arnaud (2005).

We have found that the NFW profile is a good representation of the ten observed mass profiles, and that in all cases the isothermal sphere model (i.e a profile with a core) is rejected at high confidence. In other words, we confirm the cusped nature of the Dark Matter profile, as predicted by CDM simulations

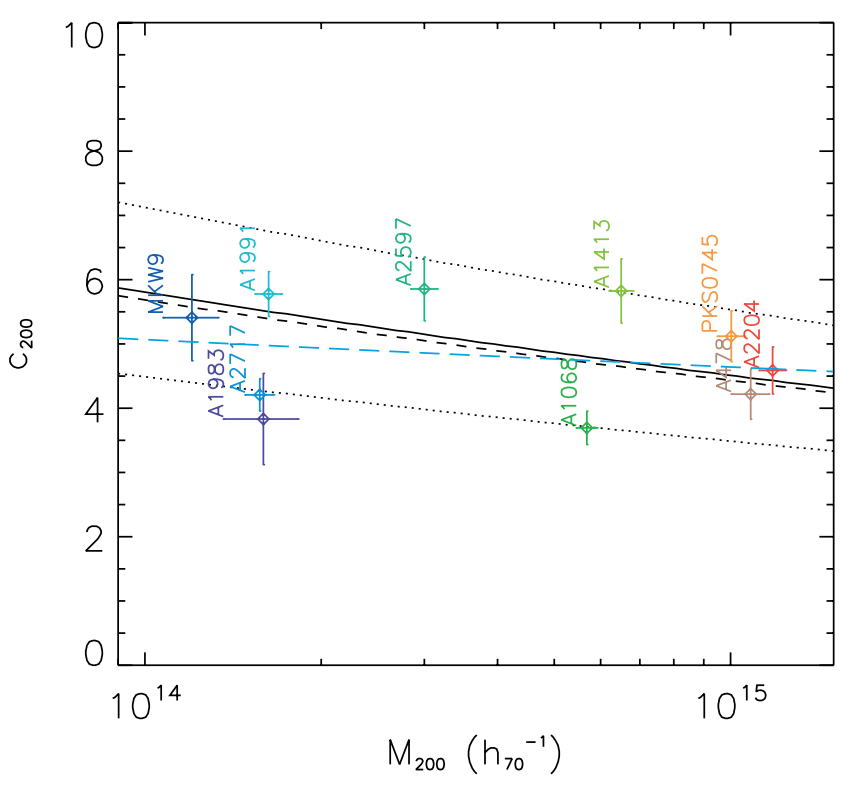

Fig. 3. Concentration parameter $c_{200}$ versus the cluster mass $M_{200}$. The solid line represents the variation of $c_{200}$ for clusters at $z=0$ from the numerical simulations of Dolag et al. (2004). The dotted lines are the standard deviation associated with this relation. The dashed line represents the same relation at a redshift of $z=0.15$ (the maximum redshift for our sample). The long-dashed line stands for our best fit of the $c_{200}-M_{200}$ (see text).

of hierarchical structure formation, over the temperature/mass range of the present sample. The mass profile shape is close to universal, again as predicted, with a dispersion of less than $15 \%$ at $0.1 R_{200}$ in the scaled mass profiles. The shape is quantitatively consistent with theoretical predictions. The variation of the observed concentration parameters with mass is in line with the predictions, taking into account the measurement errors and the expected intrinsic scatter. However, our sample is still too small to draw any firm conclusions on the exact form of the $c(M)$ relation. Taken together, our results provide further strong evidence in favour of the Cold Dark Matter cosmological scenario, and show that the physics of the Dark Matter collapse is well understood.

The exact inner slope of the CDM distribution in haloes remains an important theoretical issue (Diemand et al. 2004; Navarro et al. 2004). Few of our mass profiles have the required radial coverage and statistical quality in the central parts to allow us to firmly distinguish between an NFW-type profile and other types of cusped DM profile (e.g., the mass profile of A478). We caution also that the very central parts of clusters are regions of complex phenomena (hot bubbles, ghost cavities, cold fronts, interaction with the central galaxy, etc) which are still not well understood. Their effect on the ICM may challenge the hypothesis of hydrostatic equilibrium, and therefore the reliability of the X-ray mass estimate.

Observations are also needed of the outskirts of clusters. To date, those regions are largely unknown to observers, and can only be investigated with numerical simulations. Study of these regions is clearly needed to advance our understanding of structure formation and evolution. 
Acknowledgements. The authors thank the anonymous referee for remarks and comments. This research has made use of the XMM-Newton archives and of the SIMBAD database, operated at CDS, Strasbourg, France. E.P. acknowledges the financial support of CNES (the French space agency). G.W.P. acknowledges funding from a Marie Curie Intra-European Fellowship under the FP6 programme (Contract No. MEIF-CT-2003-500915). The authors thank Doris Neumann for interesting discussions and Nabila Aghanim for her help in the early analysis of A2204.

\section{References}

Allen, S. W., Schmidt, R. W., \& Fabian, A. C. 2001, MNRAS, 328, L37

Allen, S. W., Schmidt, R. W., Fabian, A. C., \& Ebeling, H. 2003, MNRAS, 342, 287

Arabadjis, J. S., Bautz, M. W., \& Garmire, G. P. 2002, ApJ, 572, 66

Arnaud, K. A. 1996, in Astronomical Data Analysis Software and Systems V, ASP Conf. Ser., 101, 17

Arnaud, M., Neumann, D. M., Aghanim, N., et al. 2001, A\&A, 365, L80

Arnaud, M., Aghanim, N., \& Neumann, D. M. 2002a, A\&A, 389, 1

Arnaud, M., Majerowicz, S., Lumb, D., et al. 2002b, A\&A, 390, 27

Arnaud, M., Pratt, G. W., \& Pointecouteau, E. 2004, Mem. Soc. Astron. It., 75, 529

Bertschinger, E. 1985, ApJS, 58, 39

Bullock, J. S., Kolatt, T. S., Sigad, Y., et al. 2001, MNRAS, 321, 559

Buote, D. A., \& Lewis, A. D. 2004, ApJ, 604, 116

Buote, D. A. 2004, IAU Symp., 220, 149

Chen, Y., Ikebe, Y., \& Böhringer, H. 2003, A\&A, 407, 41

David, L. P., Nulsen, P. E. J., McMamara, B. R., et al. 2001, ApJ, 557, 546

De Grandi, S., \& Molendi, S. 1999, A\&A, 351, L45

De Grandi, S., \& Molendi, S. 2002, ApJ, 567, 163

Diemand, J., Moore, B., \& Stadel, J. 2004, MNRAS, 353, 624

Dolag, K., Bartelmann, M., Perrotta, F., et al. 2004, A\&A, 416, 853

Ghizzardi, S. 2001, EPIC-MCT-TN-011 (XMM-SOC-CAL-TN0022)
Ghizzardi, S. 2002, EPIC-MCT-TN-012

Hartmann, D., \& Burton, W. B. 1999, VizieR Online Data Catalog, 8054, 0

Hicks, A. K., Wise, M. W., Houck, J. C., \& Canizares, C. R. 2002, ApJ, 580, 763

Irwin, J. A., \& Bregman, J. N. 2000, ApJ, 538, 543

Kolokotronis, V., Basilakos, S., Plionis, M., \& Georgantopoulos, I. 2001, MNRAS, 320, 49

Lewis, A. D., Buote, D. A., \& Stocke, J. T. 2003, ApJ, 586, 135

Lumb, D. H., Warwick, R. S., Page, M., \& De Luca, A. 2002, A\&A, 389, 93

McNamara, B. R., Wise, M. W., Nulsen, P. E. J., et al. 2001, ApJ, 562, L149

Markevitch, M., Forman, W. R., Sarazin, C. L., \& Vikhlinin, A. 1998, ApJ, 503, 77

Moore, B., Quinn, T., Governato, F., Stadel, J., \& Lake, G. 1999, MNRAS, 310, 1147

Navarro, J. F., Frenk, C. S., \& White, S. D. M. 1997, ApJ, 490, 493

Navarro, J. F., Hayashi, E., Power, C., et al. 2004, MNRAS, 349, 1039

Neumann, D., \& Böhringer, H. 1995, A\&A, 301, 865

Neumann, D. M., \& Arnaud, M. 2001, A\&A, 373, L33

Pointecouteau, E., Arnaud, M., Kaastra, J., \& de Plaa, J. 2004, A\&A, 423, 33

Pratt, G. W., \& Arnaud, M. 2002, A\&A, 394, 375

Pratt, G. W., \& Arnaud, M. 2003, A\&A, 408, 1

Pratt, G. W., \& Arnaud, M. 2005, A\&A, 429, 791

Read, A. M., \& Ponman, T. J. 2003, A\&A, 409, 395

Rowley, D. R., Thomas, P. A., \& Kay, S. T. 2004, MNRAS, 352, 508

Sanders, J. S., Fabian, A. C., \& Taylor, G. B. 2004, MNRAS, 356, 1022

Sharma, M., McNamara, B. R., Nulsen, P. E. J., et al. 2004, ApJ, 613, 180

Sun, M., Jones, C., Murray, S. S., et al. 2003, ApJ, 587, 619

Vikhlinin, A., Forman, W., \& Jones, C. 1999, ApJ, 525, 47

Wise, M. W., McNamara, B. R., \& Murray, S. S. 2004, ApJ, 601, 184

Wechsler, R. H., Bullock, J. S., Primack, J. R., Kravtsov, A. V., \& Dekel, A. 2002, ApJ, 568, 52

Zhang, Y.-Y., Finoguenov, A., Böhringer, H., et al. 2004, A\&A, 413, 49 
E. Pointecouteau et al.: The structural and scaling properties of nearby galaxy clusters. I., Online Material p 1

\section{Online Material}




\section{Appendix A: Cluster specifics}

For each cluster in our sample, we searched the literature for special features such as ICM bubbles, ghost cavities, cold fronts or other phenomena that could disturb the relaxed structure of the intra-cluster medium and therefore invalidate out hypothesis of spherical symmetry and hydrostatic equilibrium. We particularly investigated existing Chandra observations of central substructure in clusters, capitalising on its high spatial resolution. We used this information to check our mass profiles and if needed to exclude one (or more) point(s) containing such substructure. Details and notes are now given for individual clusters.

A478 - From the Chandra observations, Sun et al. (2003) reported the presence of X-ray cavities within the 15 central kpc. The recent XMM-Newton observation of A478 (Pointecouteau et al. 2004) excluded this inner area for the mass profile computation. We used the published mass profile of Pointecouteau et al. (2004).

A1068 - We chose this cluster for its intermediate temperature of about 4-5 keV. The data for A1068 were retrieved from the XMM-Newton archive. According to the Chandra observation detailed in Wise et al. (2004), no special inner structure has been seen.

A1413 - This cluster has been studied in detail by Pratt \& Arnaud (2002). Among the sample it is the only hot cluster without a cool core, and the only cluster with a firmly observed decrease in temperature at large radii. It is also the cluster observed up to the largest radius $\left(0.7 R_{200}\right)$. The existing (public) Chandra observation of this cluster has a too low an exposure time to show clearly whether there is structure in the cluster centre.

A1983 - Pratt \& Arnaud (2003) published an extensive study of the XMM-Newton observations for this cluster. We used all the mass points published by those authors. No Chandra $\mathrm{X}$-ray observation is yet reported for this cluster.

A1991 - Sharma et al. (2004), using Chandra, have reported low energy knots $(k T \sim 0.8 \mathrm{keV})$ located at $\sim 10 \mathrm{kpc}$ from the cluster centre. The first bin of the temperature (and thus mass) profile, used by Pratt \& Arnaud (2005) in their XMM-Newton study of A1991, extends up to 38 arcsec. The knot structure seen by Chandra is included in this bin and is therefore diluted within the cluster emission. Keeping or removing the first point in our mass analysis leads to mass modelling results which are compatible at a $1 \sigma$ level. The NFW fit being slightly better when using all the points, we chose to use the whole mass profile published by Pratt \& Arnaud (2005).

A2204 - This is a very well relaxed cluster at a redshift of $z=0.1523$ showing a very peaked emission profile toward the centre. The cluster hosts a very strong radio source at its centre, and recently Sanders et al. (2004) have pointed out complex structure in the very central part of the cluster using the Chandra satellite. In addition, as discovered in many other clusters, two cold fronts were observed at radii of about 30 and $80 \mathrm{kpc}$. While we computed the mass profile down to small radii for this cluster, we excluded in the mass analysis the two inner annuli, the outer radius of which extend up to $109 \mathrm{kpc}$, in order to avoid invalidating the assumption of hydrostatic equilibrium.

A2717 - Another poor cluster from Pratt \& Arnaud (2005). No high resolution X-ray imaging has been performed to date on this cluster. However, a work based on ROSAT/PSPC and APM optical data aiming to find substructure in a sample of clusters (Kolokotronis et al. 2001), reported substructure in the optical for A2717. It is undetectable in this X-ray observation. No significant change occurring when excluding the central point from the fit, we used the entire computed mass profile.

A2597 - This cluster was taken from the XMM-Newton archive, and was chosen because of its intermediate temperature of about 3-4 keV. A2597 has been studied with the Chandra satellite, exhibiting ghost cavities in its centre at a radius of about $30 \mathrm{kpc}$ (McNamara et al. 2001). Moreover, the Chandra SB profile and temperature profile seems to be quite disturbed up to a few tens of kpc. We therefore chose to ignore the two first data points of our mass profile and to only keep points with inner radii larger than $50^{\prime \prime}$.

MKW9 - This cluster is the least regular of the three cool systems recently studied by Pratt \& Arnaud (2005). However, lacking any external information on its central structure we use their published mass profile.

PKS 0745-191 - A previous analysis of XMM-Newton data was published by Chen et al. (2003). However, we decided to reprocess the whole dataset to keep the data processing in our sample coherent. The data for this cluster are strongly disturbed by important flaring periods. After flare cleaning, we kept only the MOS data, the EPN data being still very noisy. Our temperature profile is in total agreement with that of Chen et al. (2003), and also with the measurement by BeppoSAX at large radii (De Grandi \& Molendi 1999). Note that the overall normalisation of our temperature profile is somehow lower to that obtained by Hicks et al. (2002) with the Chandra satellite. Indeed, these authors obtained a plateau temperature of about $10.5 \mathrm{keV}$ above $200 \mathrm{kpc}$ from the centre against our value of $\sim 8 \mathrm{keV}$. Regarding the SB profile from the Chandra observation, which exhibits a sharp central cusp (mainly due to the cD galaxy), we improved the fit of the mass profile by excluding our central bin which extends up to $34^{\prime \prime}$. 